\title{
Perilipin Overexpression in White Adipose Tissue Induces a Brown Fat-Like Phenotype
}

\author{
Takashi Sawada ${ }^{1}$, Hideaki Miyoshi ${ }^{1 *}$, Kohei Shimada ${ }^{2}$, Akira Suzuki ${ }^{3}$, Yuko Okamatsu-Ogura ${ }^{2}$, James W. \\ Perfield II ${ }^{4}$, Takuma Kondo ${ }^{1}$, So Nagai ${ }^{1}$, Chikara Shimizu ${ }^{1}$, Narihito Yoshioka ${ }^{1}$, Andrew S. Greenberg ${ }^{5}$, \\ Kazuhiro Kimura ${ }^{2}$, Takao Koike ${ }^{1}$
}

1 Department of Internal Medicine II, Hokkaido University Graduate School of Medicine, Sapporo, Japan, 2 Department of Biomedical Sciences, Hokkaido University Graduate School of Veterinary Medicine, Sapporo, Japan, 3 Department of Pathology, KKR Sapporo Medical Center, Sapporo, Japan, 4 Department of Nutrition and Exercise Physiology and Food Science, University of Missouri, Columbia, Missouri, United States of America, $\mathbf{5}$ Jean Mayer United States Department of Agriculture Human Nutrition Research Center on Aging, Tufts University, Boston, Massachusetts, United States of America

\begin{abstract}
Background: Perilipin A (PeriA) exclusively locates on adipocyte lipid droplets and is essential for lipid storage and lipolysis. Previously, we reported that adipocyte specific overexpression of PeriA caused resistance to diet-induced obesity and resulted in improved insulin sensitivity. In order to better understand the biological basis for this observed phenotype, we performed additional studies in this transgenic mouse model.

Methodology and Principal Findings: When compared to control animals, whole body energy expenditure was increased in the transgenic mice. Subsequently, we performed DNA microarray analysis and real-time PCR on white adipose tissue. Consistent with the metabolic chamber data, we observed increased expression of genes associated with fatty acid $\beta$ oxidation and heat production, and a decrease in the genes associated with lipid synthesis. Gene expression of Pgc1a, a regulator of fatty acid oxidation and Ucp1, a brown adipocyte specific protein, was increased in the white adipose tissue of the transgenic mice. This observation was subsequently verified by both Western blotting and histological examination Expression of RIP140, a regulator of white adipocyte differentiation, and the lipid droplet protein FSP27 was decreased in the transgenic mice. Importantly, FSP27 has been shown to control gene expression of these crucial metabolic regulators. Overexpression of PeriA in 3T3-L1 adipocytes also reduced FSP27 expression and diminished lipid droplet size.

Conclusions: These findings demonstrate that overexpression of PeriA in white adipocytes reduces lipid droplet size by decreasing FSP27 expression and thereby inducing a brown adipose tissue-like phenotype. Our data suggest that modulation of lipid droplet proteins in white adipocytes is a potential therapeutic strategy for the treatment of obesity and its related disorders.
\end{abstract}

Citation: Sawada T, Miyoshi H, Shimada K, Suzuki A, Okamatsu-Ogura Y, et al. (2010) Perilipin Overexpression in White Adipose Tissue Induces a Brown Fat-Like Phenotype. PLoS ONE 5(11): e14006. doi:10.1371/journal.pone.0014006

Editor: Massimo Federici, University of Tor Vergata, Italy

Received May 4, 2010; Accepted October 26, 2010; Published November 16, 2010

Copyright: (c) 2010 Sawada et al. This is an open-access article distributed under the terms of the Creative Commons Attribution License, which permits unrestricted use, distribution, and reproduction in any medium, provided the original author and source are credited.

Funding: This work was supported by grants from the Japanese Ministry of Education, Culture, Sports, Science, and Technology Foundation (http://www.mext. go.jp/), Takeda Science Foundation (http://www.takeda-sci.or.jp/), Kanae Foundation for the Promotion of Medical Science (http://kanae.sanofi-aventis.co.jp/), and Kissei Pharmaceutical Foundation (http://www.kissei.co.jp/). The funders had no role in study design, data collection and analysis, decision to publish, or preparation of the manuscript.

Competing Interests: The authors have declared that no competing interests exist.

*E-mail: hmiyoshi@med.hokudai.ac.jp

\section{Introduction}

The metabolic syndrome is an accumulation of risk factors of cerebrovascular and cardiovascular disease such as diabetes, dyslipidemia and hypertension [1]. Increased visceral fat and elevated lipolysis cause dysfunction of various organs and abnormal production of adipokines [2]. Therefore, when considering the pathophysiology of the metabolic syndrome, it is extremely important to understand the mechanisms of lipid storage and release (lipolysis) in adipocytes. Within adipocytes, triglyceride is predominately stored within lipid droplets that are surrounded by a phospholipid monolayer containing various lipid droplet proteins. These proteins belong to the PAT family which contains Perilipin, ADRP/adipophilin, TIP47, MLDP (OXPAT/ LSD5) and S3-12 which all have homology in their N-terminal sequence [3]. Perilipin (Peri) is the predominant protein present on the surface of lipid droplets in fat cells of white/brown adipose tissue and steroid producing cells [4]. Perilipin A (PeriA) is the most abundant adipocyte lipid phosphoprotein, which is activated by protein kinase A (PKA) and is considered to play a central role in regulating lipid metabolism in adipocytes by controlling various proteins [5]. Ablation of PeriA from white adipose tissue (WAT) causes dysregulation of adipocyte lipid storage characterized by increased basal lipolysis and decreased PKA-stimulated lipolysis and results in a dramatic reduction in WAT mass [6,7]. The role of PeriA in WAT is to suppress lipolysis in the absence of PKA stimulation, and enhance lipolysis $(\sim 100$ fold $)$ with PKA stimulation $[8,9]$.

Recently, fat specific protein 27 (FSP27 or Cidec) was identified as a protein which localizes on the surface of lipid droplets in white 
adipocytes and contributes to energy storage by promoting the formation of unilocular lipid droplets [10,11]. FSP27 deficiency dramatically reduced WAT mass and induced a brown adipocytelike morphology in the WAT via reducing the factors inhibiting brown adipocyte differentiation such as receptor interacting protein 140 (RIP140) and increasing brown adipocyte-specific genes or key metabolic controlling factors such as PPAR coactivator $1 \alpha(\mathrm{PGC} 1 \alpha[1112]$.

Previously, we generated transgenic mice which overexpressed either human or mouse PeriA specifically in adipocytes and studied these mice in the context of obesity and lipid/glucose metabolism [13]. When challenged with a high fat diet (HFD), both human and mouse PeriA Tg mice gained less weight, and had reduced WAT mass, though their food intake was similar to that of wild type (WT) mice. In this manuscript, we performed further studies in this human PeriA Tg mouse model to investigate the mechanisms of obesity-resistance and metabolic changes.

\section{Results}

Increased oxygen consumption and energy expenditure in PeriA Tg mice

Consistent with our previous study [13], body weight and subcutaneous and gonadal WAT mass were reduced in HFD-fed $\mathrm{Tg}$ mice as compared to HFD-fed WT mice (data not shown). With regard to energy metabolism, whole-body oxygen consumption rate $\left(\mathrm{VO}_{2}\right)$ in HFD-fed human PeriA Tg mice was markedly higher than that of WT controls (Figure 1A). Twenty-four-hour oxygen consumption and energy expenditure were significantly increased in Tg mice maintained on a HFD and this difference was maintained after correction for differences in fat pad mass compared with the corresponding values for WT mice (Figure 1B, C). Furthermore, we measured oxygen consumption of white adipocytes isolated from PeriA Tg and wild-type mice to examine their mitochondrial function. Consistent with our metabolic chamber data in vivo, both basal and norepinephrine (NE) stimulated oxygen consumption tended to be increased in white adipocytes isolated from PeriA Tg mice (Figure S1).

\section{Up-regulation of brown fat associated transcriptional factors, and down-regulation of lipid synthesis genes in WAT of Tg mice}

Subsequently we employed DNA microarray analysis on WAT from $\mathrm{Tg}$ and WT mice. We observed increased expression of genes associated with fatty acid $\beta$-oxidation and heat production, and a decrease in the genes involved in lipid synthesis (Table 1, Figure 2). To validate these microarray data, we next performed real-time PGR on selected gene targets. Consistent with the metabolic chamber data, we confirmed significant increases in the expression of Cpt1 (WT $1.00 \pm 0.15$ vs. Tg 2.13 $\pm 0.81, p=0.049$ ) and $M c d$ (WT $1.00 \pm 0.18$ vs. $\mathrm{Tg} 1.87 \pm 0.26, p=0.021)$ that are involved in fatty acid $\beta$-oxidation and energy expenditure (Figure $3 \mathrm{~A}$ ). On the other hand, we observed significant decreases in the expression of the lipogenic genes $S c d 1$ (WT $1.00 \pm 0.33$ vs. $\mathrm{Tg} 0.10 \pm 0.06$, $p=0.014)$, Dgat1 (WT $1.00 \pm 0.59$ vs. Tg 0.15 $\pm 0.07, p=0.018), L p l$ (WT $1.00 \pm 0.24$ vs. Tg $0.25 \pm 0.02, p=0.002$ ) and Fas (WT $1.00 \pm 0.46$ vs. $\operatorname{Tg} 0.08 \pm 0.04, p=0.016)$. Similarly, the expression of Scd2, Dgat2, Srebp1c and Acc tended to be decreased (Figure 3B). Notably, expression of Rip140 which is involved in the differentiation of white adipocytes was remarkably decreased (WT $1.00 \pm 0.43$ vs. $\mathrm{Tg} 0.02 \pm 0.01, p=0.002)$, while $P g c 1 \alpha$, a regulator of mitochondrial biogenesis, was significantly increased (WT $1.00 \pm 0.42$ vs. $\mathrm{Tg} 16.59 \pm 8.66, p=0.003$ ) (Figure 3C).

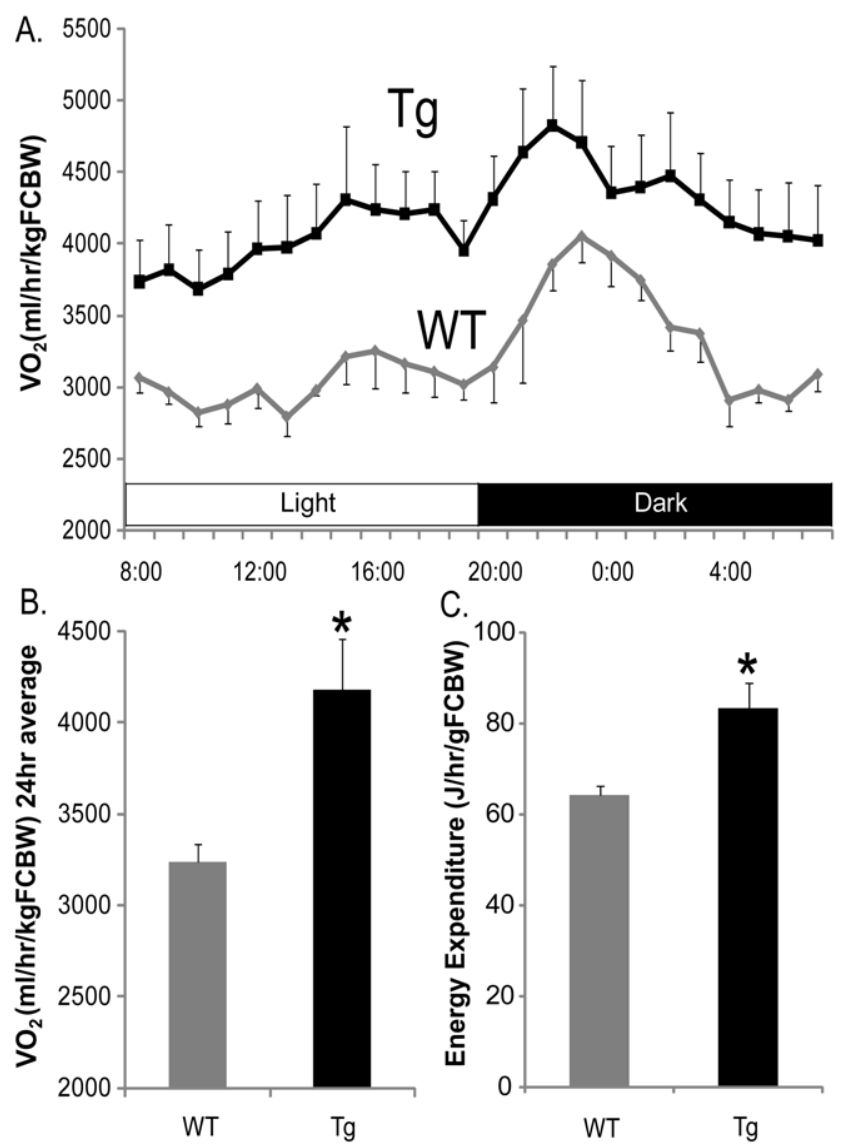

Figure 1. Oxygen consumption and energy expenditure. (A) Whole-body oxygen consumption rate during a 12-hour dark/12-hour light cycle for 30-week-old mice fed a high fat diet ( $n=7$ for WT; $n=7$ for $\mathrm{Tg}$ ). (B) The average value during the 24-hour period. (C) Energy expenditure during 24-hour period in the experiment. FCBW: fatcorrected body weight $=$ body weight - (mass of subcutaneous and perigonadal white adipose tissue). Data are mean \pm SEM. ${ }^{*}, p<0.05$. doi:10.1371/journal.pone.0014006.g001

\section{Ectopic expression of UCP1, a brown adipose tissue} specific protein, in white adipose tissue of PeriA Tg mice

Uncoupling protein-1 (UCP1) functions to uncouple oxidative phosphorylation and converts the proton gradient energy to heat to maintain body temperature. It is thought to be expressed exclusively in brown adipocytes, and is considered a specific marker of brown adipocytes [14]. We confirmed the ectopic expression of $U_{c p 1}$ in the WAT of Tg mice by microarray and real-time PGR (Table 1, Figure 4A). UCP1 protein expression in the WAT of $\mathrm{Tg}$ mice was subsequently verified by both western blotting (Figure 4B) and histological immunostaining (Figure 4G). However, despite being increased, the UCP1 expression in WAT of $\mathrm{Tg}$ mice was much less than was present in brown adipose tissue (Figure S2). Histological analysis revealed that UCP1 was expressed in the smaller adipocytes in the WAT of Tg mice, but not in the WAT of WT mice. In addition, the previously reported increase in CPT1 gene expression (Figure 3A) in WAT of Tg mice was also confirmed by Western blotting (Figure 4B).

\section{The abundance of the lipid droplet protein FSP27 is} reduced in the WAT of PeriA Tg mice

The mRNA expression of the lipid droplet protein Fsp27 was significantly decreased in the WAT of PeriA Tg mice compared 
Table 1. Gene expression of WAT in microarray analysis.

\begin{tabular}{|c|c|c|}
\hline Gene/protein name (symbol) & UniGene ID & Fold change \\
\hline \multicolumn{3}{|l|}{ Adipokines } \\
\hline Adiponectin receptor 2 (Adipor2) & Mm.291826 & 1.52 \\
\hline Adiponectin receptor 1 (Adipor1) & Mm.259976 & 1.15 \\
\hline Adiponectin (Adipoq) & Mm.3969 & 1.00 \\
\hline Lipoprotein lipase (Lpl) & Mm.1514 & -1.07 \\
\hline Monocyte chemotactic protein-1; chemokine (C-C motif) ligand 2 (Ccl2) & Mm.290320 & -1.23 \\
\hline Leptin (Lep) & Mm.277072 & -1.52 \\
\hline \multicolumn{3}{|l|}{ Adipocyte differentiation } \\
\hline PRD1-BF1-RIZ1 homologous domain containing 16 (Prdm16) & Mm.257785 & 1.41 \\
\hline Peroxisome proliferator activated receptor gamma, coactivator 1 alpha (Pgargc1a) & Mm.259072 & 1.23 \\
\hline \multicolumn{3}{|l|}{ Fatty acid oxidation and thermogenesis } \\
\hline Uncoupling protein 1 (Ucp1) & Mm.4177 & 64.00 \\
\hline Carnitine parmitoyltransferase 1 (Cpt1) & Mm.34881 & 3.25 \\
\hline Carnitine/acylcarnitine translocase (Cac) & Mm.29666 & 1.23 \\
\hline 3-Ketoacyl-CoA thiolase B (Kat) & Mm.30417 & 1.15 \\
\hline \multicolumn{3}{|l|}{ Lipid synthesis } \\
\hline Stearoyl-CoA desaturase $1(\mathrm{Scd} 1)$ & Mm.267377 & -1.32 \\
\hline Diacylglycerol acyltransferase 2 (Dgat2) & Mm.180189 & -1.74 \\
\hline Stearoyl-CoA desaturase $2(\mathrm{Scd} 2)$ & Mm.193096 & -1.87 \\
\hline \multicolumn{3}{|l|}{ Electron transport chain } \\
\hline cytochrome c oxidase, subunit VIla 1 (Cox7a1) & Mm.12907 & 3.03 \\
\hline cytochrome c oxidase, subunit VIIllb (Cox8b) & Mm.3841 & 2.64 \\
\hline ATP synthase, $\mathrm{H}+$ transporting, $\mathrm{F} 1$ gamma 1 (Atp5g1) & Mm.258 & 1.07 \\
\hline
\end{tabular}

doi:10.1371/journal.pone.0014006.t001

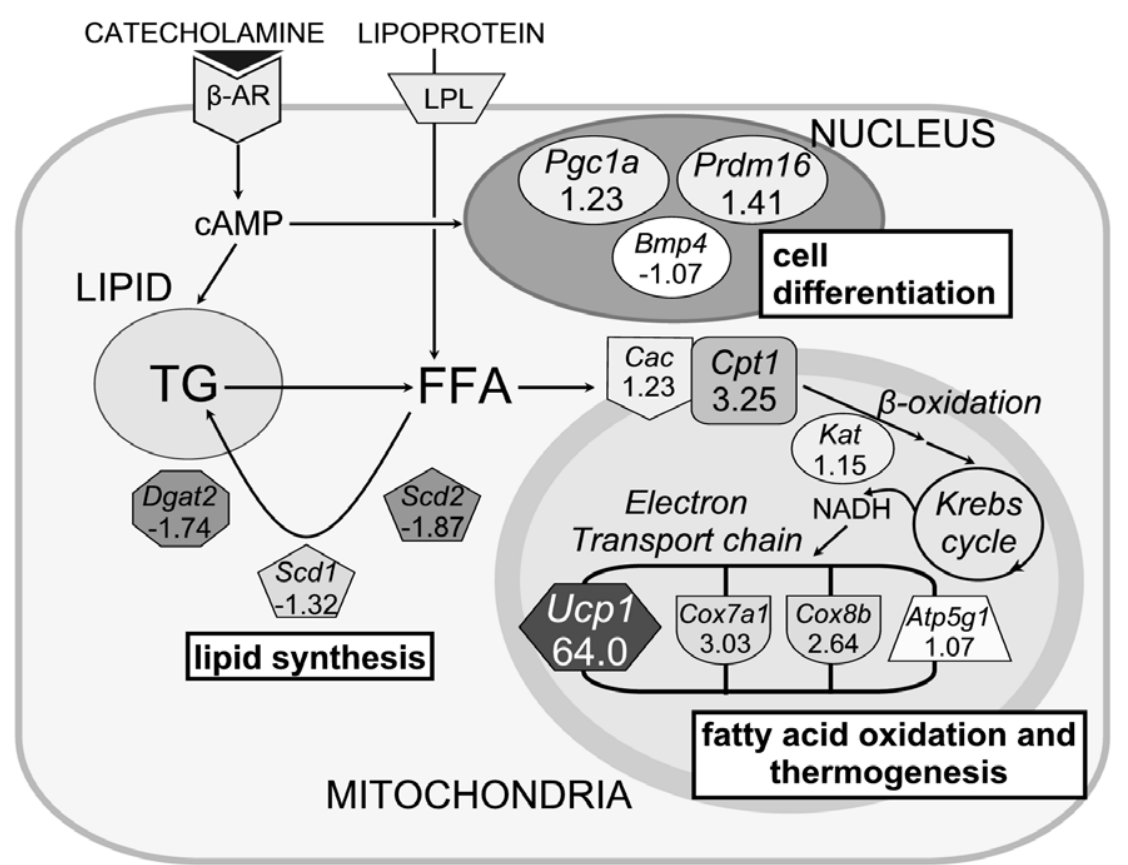

Figure 2. DNA microarray analysis. Metabolic pathways altered in WAT of Tg mice: cell differentiation, lipid synthesis, fatty acid oxidation, and thermogenesis. Fold changes in transcript levels are noted beneath the gene symbols. Atp5g1: ATP synthase, H+ transporting, F1 gamma 1, Bmp: bone morphogenic protein, Cac: carnitine/acylcarnitine translocase, Cox: cytochrome c oxidase, Cpt: carnitine palmitoyl transferase, Dgat: diacylglycerol acyltransferase, Kat: 3-ketoacyl-CoA thiolase, Pgc1a: peroxisome proliferator activated receptor gamma coactivator-1 alpha, Prdm16: PRD1-BF1-RIZ1 homologous domain containing 16, Scd: stearoyl-CoA desaturase.

doi:10.1371/journal.pone.0014006.g002 
A.
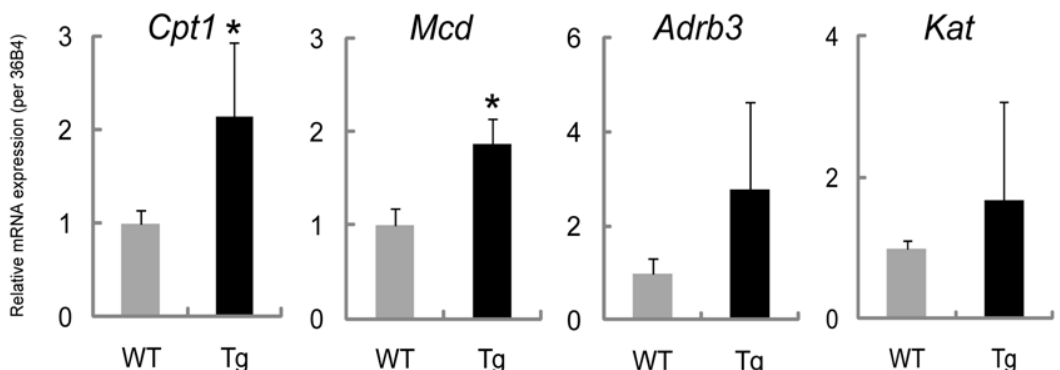

B.
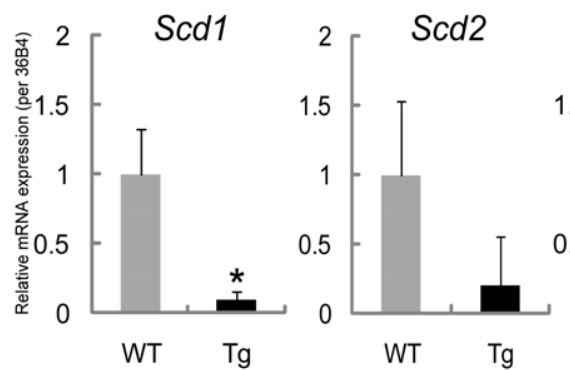

WT Tg

WT $\quad \mathrm{Tg}$
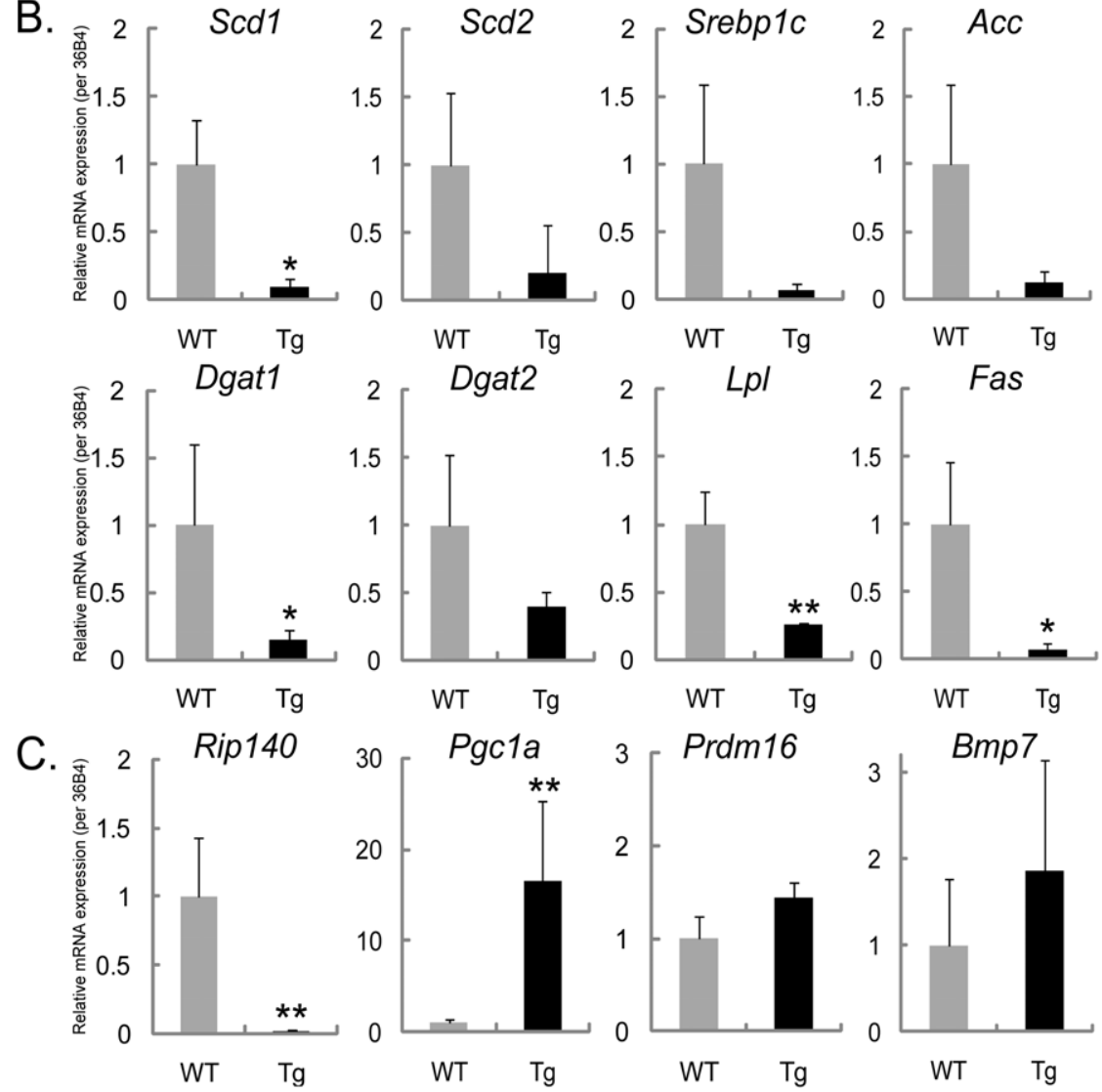

Figure 3. Quantitative PCR. Quantitative real-time PCR analysis of the expression of genes in WAT of 30-week-old WT and Tg mice fed a high fat diet, related to (A) fatty acid oxidation and thermogenesis, (B) lipid synthesis, and (C) cell differentiation to white/brown adipocytes. Data were normalized by the amount of $36 B 4 \mathrm{mRNA}$ and expressed relative to the corresponding value for WAT of WT mice; Data are mean \pm SEM $(n=9)$. ${ }^{*}, p<0.05 ;{ }^{* *}, p<0.01$. Acc: acetyl-CoA carboxylase, Adrb3: beta-3 adrenergic receptor, Fas: fatty acid synthease, Lpl: lipoprotein lipase, Mcd: malonylCoA decarboxylase, Srebp1c: sterol regulatory element binding protein $1 c$. doi:10.1371/journal.pone.0014006.g003

with WT mice (WT $1.00 \pm 0.22$ vs. Tg $0.18 \pm 0.05, p=0.0007$ ) (Figure 5A). Similarly, protein expression of FSP27 and RIP140 were decreased in $\mathrm{Tg}$ mice (Figure $5 \mathrm{~B}$ ). As would be expected, the protein content of PeriA was clearly increased in the WAT of Tg mice when compared to control (Figure 5B).

\section{Expression of FSP27 was directly attenuated by PeriA overexpression in vitro}

To further study the relationship between the expression of PeriA and FSP27 in adipocytes, we used an adenoviral system to overexpress human PeriA in cultured 3T3-L1 adipocytes. Overexpression of PeriA resulted in a dramatic reduction in lipid droplet size in cultured 3T3-L1 adipocytes (Figure 6A). This reduction in lipid droplet size was accompanied by a decrease in FSP27 protein expression (Figure 6B). These observations are consistent with our in vivo data from the WAT of the PeriA Tg mice. FSP27 locates on the surface of lipid droplets much like PeriA and functions to promote the formation of unilocular lipid droplet in white adipocytes [11]. Interestingly, our western blot data reveal that there is an inverse relationship between expression of PeriA and FSP27 (Figure 6B). Furthermore, we analyzed the mRNA expression level for genes involved in adipocyte differentiation, lipid synthesis, and fatty acid $\beta$-oxidation in PeriA-overexpressed 3T3-L1 samples. Consistent with our observations in the WAT of Tg mice, we observed downregulation of Rip140 and up-regulation of Pgc1a. Also, we observed increased expression of genes associated with fatty acid oxidation and mitochondrial biogenesis, and a decrease in the genes associated with lipid synthesis (Figure 6C). 


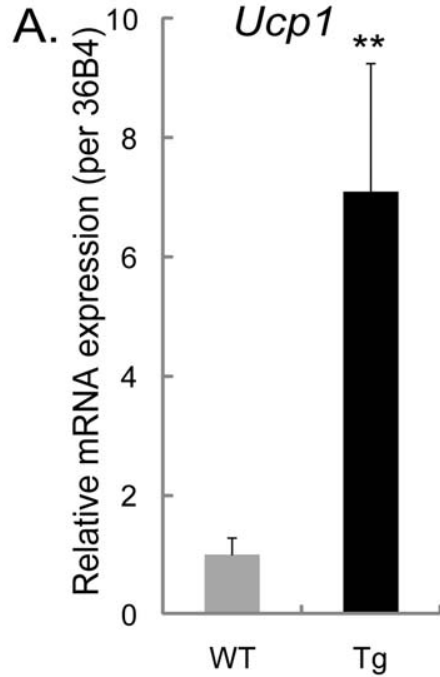

C.

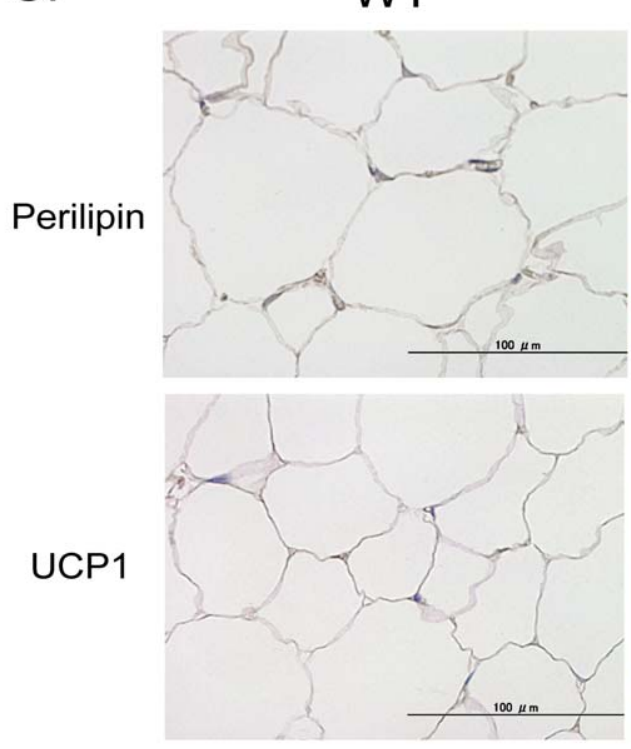

B.
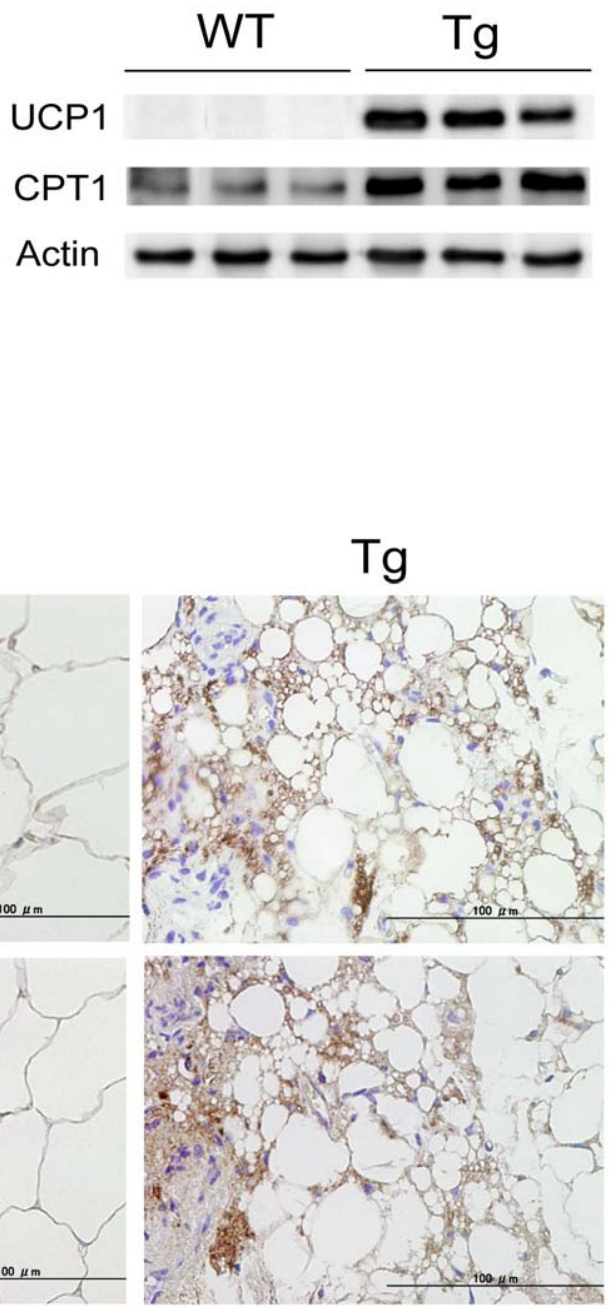

Figure 4. Ectopic expression of UCP1. Ectopic expression of UCP1 in WAT of 30-week-old PeriATg mice fed a chow diet. (A) Quantitative realtime PCR analysis of Ucp 1 mRNA expression in WAT of WT and Tg mice. Data are mean \pm SEM $(n=7)$. **, $p<0.01$. (B) Western blot analysis showing UCP1 or CPT1 protein expression in WT and Tg mice. (C) Innunohistochemistry of PeriA or UCP1 in WAT of WT and Tg mice. Original magnification, $\times 400$.

doi:10.1371/journal.pone.0014006.g004

\section{Discussion}

It has been previously reported that Peri knockout mice lack the ability to store lipid due to a chronic low level of lipolysis, and therefore have diminished adipose depots and are obesity-resistant $[6,7]$. We initially hypothesized that overexpression of PeriA in $\mathrm{Tg}$ mice would cause increased storage of lipid in adipose tissue and promote an obese phenotype. However, our original characterization of the PeriA Tg mice revealed that these animals had less adipose tissue and were resistant to diet-induced obesity [13]. We suggested that one mechanism underlying the resistance to HFDinduced obesity in PeriA Tg mice was an upregulation of oxidative genes in brown adipose tissue (BAT) [13]. In the current study, we further analyzed various metabolism-related proteins in WAT, and confirmed that the expression of genes related to $\beta$-oxidation and thermogenesis were increased in WAT as well as BAT. We performed a microarray to expand these initial findings. Our DNA microarray and RT-PGR data revealed an upregulation of $U_{c p} 1$, a brown adipocyte specific protein, in WAT of $\mathrm{Tg}$ mice. This increase was also confirmed by Western blot analysis. Histological analysis of the WAT demonstrated the robust expression of UCP1 protein within small adipocytes that were located between larger white adipocytes. These small adipocytes are not observed in the WAT of WT mice and appear to have a morphology more similar to brown adipocytes. While only a small number of adipocytes appear to adopt this brown-like phenotype within the WAT of Tg mice, it has recently been reported that these "brite" (brown-inwhite) adipocytes are metabolically significant as they can counteract obesity by contributing to thermogenesis [15]. The observed reduction in lipid droplet size and decreased mRNA expression of lipogenic genes in WAT of $\mathrm{Tg}$ mice might be a secondary change caused by increased fatty acid oxidation. 
A.

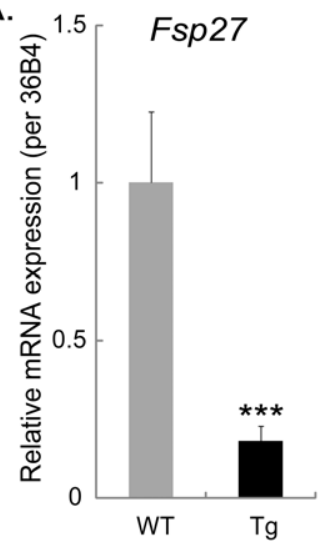

Figure 5. FSP27 expression in vivo. Alteration in the expression of lipid droplet proteins in vivo. (A) Quantitative real-time PCR analysis of Fsp27 mRNA expression in WAT of WT and Tg mice. Data are mean \pm SEM $(n=7)$. ${ }^{* * *}, p<0.001$. (B) Western blot analysis for FSP27, RIP140 and PeriA in WAT of WT and Tg mice.

doi:10.1371/journal.pone.0014006.g005

However, it is possible that overexpression of PeriA results in a direct reduction in lipid droplet size and expression of lipogenic genes as we observed a reduction in lipid droplet size when PeriA was overexpressed in culture.

In recent reports about development and differentiation of adipocytes, it is recognized that the adipoblasts, which are derived from mesenchymal stem cells and exist around capillaries in adipose tissue, will be differentiated to brown adipocytes by factors such as PRDM16, BMP7, or PGC1 $\alpha$ [16-19]. In contrast, when RIP140, a co-repressor of many nuclear receptors is present, the adipoblasts will be differentiated into white adipocytes $[16,20]$. RIP140 is also reported to interact directly with PGC1 $\alpha$ and suppress its activity [21]. These facts suggest the possibility that progenitor adipocytes could develop into brown adipocytes by changing the gene expression of these key metabolic controlling factors. In this study we observed a remarkable decrease of Rip140 mRNA and increase of Pgcla mRNA. These data suggest a potential mechanism for the observed increase in UCPl expression with PeriA overexperssion. We hypothesize that PeriA overexpression alters the expression of these key regulators of differentiation in adipoblasts which are present in WAT and causes these progenitor adipocytes to differentiate into brown adipocytes.

Overexpression of PeriA in vivo causes a reduction in FSP27 protein expression. Consistent with this observation, we demonstrated in vitro an inverse relationship exists between FSP27 protein expression and PeriA expression. This phenomenon is likely due to a simple competition theory because both proteins preferentially locate to lipid droplets. Recently, FSP27 knockout mice have been described to have a phenotype of obesity-resistance, elevated oxygen consumption, extremely reduced WAT mass and smaller white adipocytes with multilocular lipid droplets $[11,12]$. In addition, genes related to fatty acid $\beta$-oxidation and mitochondrial biosynthesis were significantly increased in FSP27 knockout mice $[11,12]$. These data are very similar to the data presented in this manuscript where we report that PeriA overexpression causes a significant reduction in FSP27 which is associated with decreased adipocyte size, upregulation of genes involved in fatty acid oxidation and a decrease in lipogenic genes. Our data suggest that the attenuation of FSP27 by overexpression of PeriA was the trigger for the down-regulation of RIP140 and up-regulation of PGC1 $\alpha$, which caused progenitor adipocytes to differentiate into brown adipocytes rather than white adipocytes. This increase in brown adipocyte-like metabolic activity resulted in an increase in energy expenditure. Although FSP27 acts as a regulator to control gene expression of crucial metabolic regulators [12], the mechanism of how the lipid droplet related structural protein FSP27 reduces the expression of transcriptional factor RIP140 has not been clarified and requires further investigation. We hypothesize that a change of intracellular environment in the adipocyte itself such as "the size of the lipid droplets" or "the composition of the lipid droplet proteins", can alter the characteristics of the adipocyte.

We revealed that PeriA overexpression results in resistance to diet-induced obesity, increased energy expenditure and reduced lipid synthesis in vivo, and that the basis for these effects was the induction of a BAT-like phenotype in WAT due to a decrease in FSP27 expression. Our data suggest that modulation of lipid droplet proteins in white adipocytes is a potential therapeutic strategy for the treatment of obesity and its related disorders.

\section{Materials and Methods}

\section{Ethics Statement}

All animal care and experimental procedures in this study were approved by Hokkaido University Animal Experiment Committee (approval number: 08-0358) and carried out according to guidelines for animal experimentation of Hokkaido University.

\section{Antibodies}

Polyclonal anti-perilipin antibody was generated as previously described [22]. Anti-UCP1 antiboby was a kind gift from Prof. Teruo Kawada (University of Kyoto, Kyoto, Japan) [23], and antiFSP27 antibody was generated by Dr. Yoshikazu Tamori, et al (Kobe University, Kobe, Japan) [11]. Anti-RIP140 antibody was purchased from Abcam plc (Cambridge, UK). Anti-actin antibody, anti-CPT1 antibody, and horseradish peroxidase-conjugated antigoat IgG were purchased from Santa Cruz Biotechnology Inc (Santa Cruz, CA). Horseradish peroxidase-conjugated anti-rabbit IgG was purchased from BIO-RAD Laboratories (Hercules, CA).

\section{Animal experiments}

We generated transgenic mice that overexpressed human PeriA using the adipocyte specific aP2 promoter/enhancer [13]. All PeriA Tg mice used for the study were female, and heterozygous for the transgene. Littermates that lacked the transgene were used as controls (WT). Relative to WT littermates, PeriA protein levels were increased 2-fold in WAT and 5-fold in BAT of Tg mice. Mice were housed in a pathogen-free barrier facility at the Institute for Animal Experimentation at Hokkaido University. All mice were housed at room temperature, maintained on a $12 \mathrm{~h}$ light/dark cycle, given free access to water, and fed a standard chow diet $(5.3 \%$ calories from fat; Oriental Yeast Co., ltd. MF, Tokyo, Japan) or a HFD (60\% calories from fat; Research Diets \#D12492, New Brunswick, NJ) for 25 weeks (from the age of 5 weeks to 30 weeks). Food intake and body weight were monitored weekly. On the day prior to tissue harvest at 30 weeks, food was removed at 21:00 h for an overnight fast. After the anesthetization with isoflurane (Abbott Japan, Tokyo, Japan), WAT from visceral (perigonadal) and subcutaneous depots were rapidly dissected out and processed for subsequent analysis.

\section{Measurement of oxygen consumption of HFD-fed Tg mice}

Oxygen consumption of 30-week-old mice in the fed condition was measured with an open-circuit-type metabolic chamber 


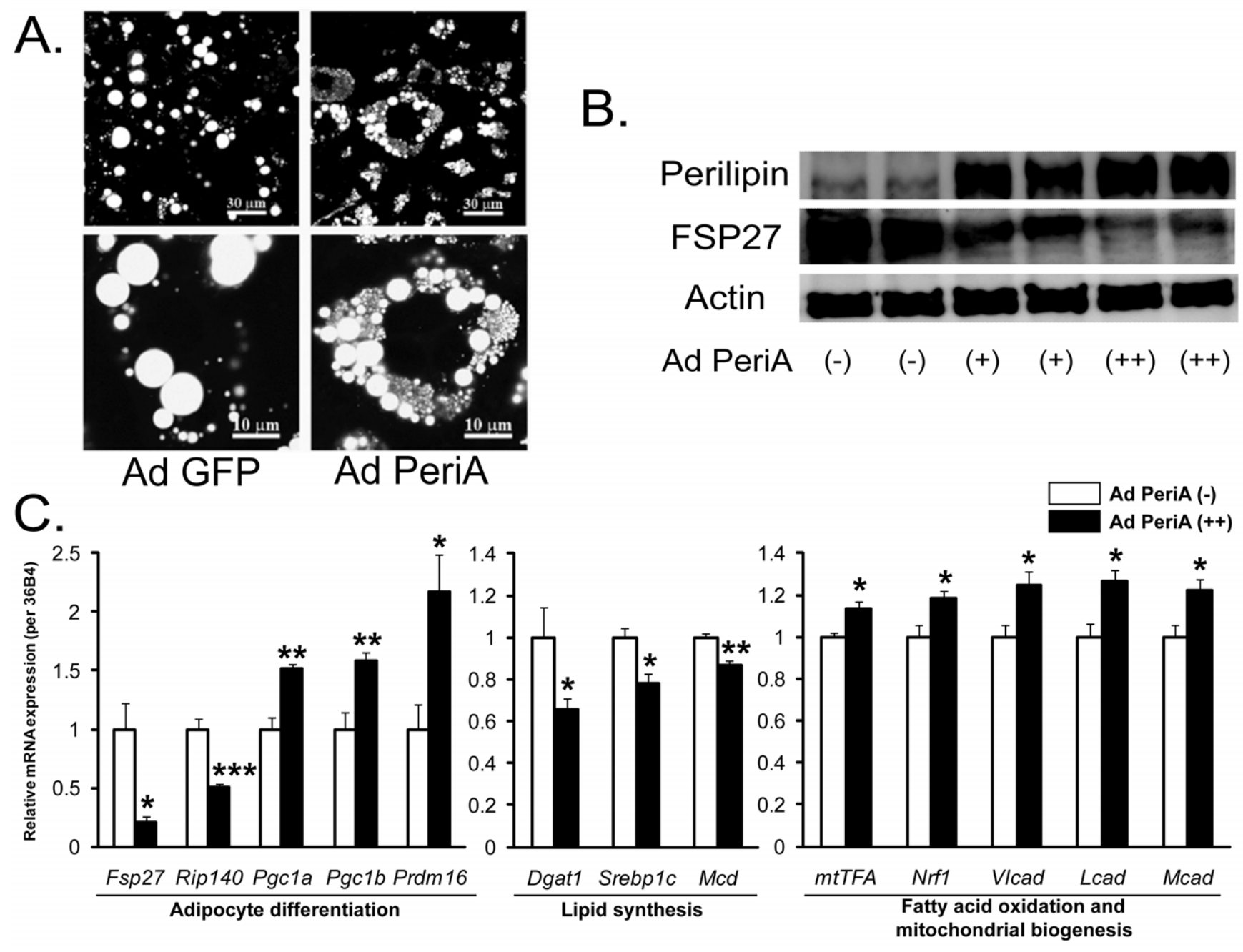

Figure 6. In vitro experiments. Change of lipid droplet size and protein expression in vitro. (A) Microscopic images of 3T3-L1 adipocytes transfected human PeriA or GFP (control) using adenoviral system plus lipofection methods. (B) Western blot analysis for lipid droplet surface proteins

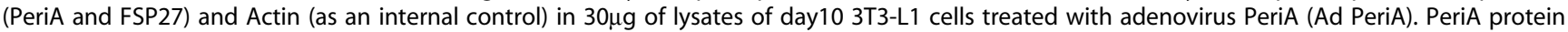
content increased dose-dependently with increasing PeriA viral titer in 3T3-L1 cells. Signs in a figure $((-),(+)$ and $(++))$ mean the amount of transfected adenovirus (none, single and double quantity). (C) Quantitative real-time PCR analysis of mRNA expression in cultured 3T3-L1 cells (white bar, Ad PeriA (-): black bar, Ad PeriA(++); Data are mean \pm SEM $\left.(n=6) .{ }^{*}, p<0.05 ;{ }^{* *}, p<0.01 ;{ }^{* * *}, p<0.001\right)$. mtTFA: mitochondrial transcription factor A, Nrf1: nuclear respiratory factor 1, Vlcad: very long-chain acyl-CoA dehydrogenase, Lcad: long-chain acyl-CoA dehydrogenase, Mcad: medium-chain acyl-CoA dehydrogenase.

doi:10.1371/journal.pone.0014006.g006

(MM202R; Muromachi Kikai, Tokyo, Japan) every 3 minutes for 48 hours (12-hour dark/12-hour light cycle) at $25^{\circ} \mathrm{C}$. Data were normalized to fat-corrected body weight (BW - the mass of subcutaneous and perigonadal white adipose tissue). Energy expenditure was calculated using the following formula: $([1.07 \times$ respiratory quotient +3.98$] \times \mathrm{VO}_{2} \times 4.2 \times 60 /$ body weight of a mouse). Measurement of oxygen consumption in isolated white adipocytes was performed as previously described [11].

\section{DNA microarray analysis}

Total RNA extraction was performed by using Ribopure Kit (Ambion, Austin, TX), according to the manufacturer's instructions. To minimize individual variation as a source of geneexpression variance, RNA samples were pooled, one pool representing the three HFD-fed $\mathrm{Tg}$ mice ( $4 \mu \mathrm{g}$ from each sample) and one representing the three HFD-fed WT mice $(4 \mu \mathrm{g}$ from each sample). RNA ( $2 \mu \mathrm{g}$ from each pool) was reverse transcribed to cDNA and tagged with biotin with one-cycle target labeling and hybridized according to the standard protocol using Mouse Genome 4302.0 array (Affymetrix), which was then washed, stained, and scanned. The results obtained from these samples were analyzed with the GeneChip Operating Software verl.4. The detection algorithm uses probe pair (includes perfect-match (PM) and mismatch (MM)) intensities to generate a detection $p$ value and assign a Present, Marginal, or Absent call. A two-step procedure determines the detection $p$-value for a given probe set: the first step calculates the discrimination score $\mathrm{R}$ for each probe pair, by a formula $\mathrm{R}=(\mathrm{PM}-\mathrm{MM}) /(\mathrm{PM}+\mathrm{MM})$. The second step tests the discrimination scores against the user-definable threshold Tau. The One-Sided Wilcoxon's Signed Rank test is the statistical method employed to generate the detection $p$-value. It assigns each probe pair a rank based on how far the probe pair discrimination score is from Tau. The detection $p$-value cut-offs, Alpha $1(\alpha 1)$ and Alpha 2 ( $\alpha 2$ ), provide boundaries for defining Present, Marginal, or Absent calls. Any $p$-value that falls below $\alpha 1$ is assigned a 'Present' call, and above $\alpha 2$ is assigned an 'Absent' call. Marginal calls are 
given to probe sets which have $p$-values between $\alpha 1$ and $\alpha 2$. The settings of quantitation parameters were: $\alpha 1=0.05, \alpha 2=0.065$, $T a u=0.015$. The genes detected to be 'Present' in the data from the microarray were passed to further analysis. Signal is a quantitative metric calculated for each probe set, which represents the relative level of expression of a transcript. The signal log ratio estimates the magnitude and direction of change of a transcript, which is calculated by $\log _{2}$ (Signal of $\mathrm{Tg} /$ Signal of WT). Differentially expressed probe sets were selected based on filtering by signal $\log$ ratio under -0.3 or over 0.3 . The microarray data files have been submitted to the Gene Expression Omnibus (GEO) and the accession number is GSE21754.

\section{Quantitative PCR}

For each gene that was determined to have expression differences in the DNA microarray, we evaluated actual differences by real-time PCR. cDNA was synthesized from $0.5 \mu \mathrm{g}$ of total RNA (High Capacity RNA-to-cDNA kit, Applied Biosystems, Warrington, UK). Real-time PCR was performed in

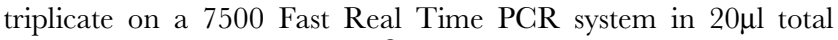
volume reactions using SYBR ${ }^{\circledR}$ Green PCR Master Mix (Applied Biosystems, Warrington, UK). Primers were designed using Primer Express. Data were analyzed by comparative critical threshold $(\mathrm{Ct})$ method [24] and normalized to an endogenous control gene (36B4: acidic ribosomal phosphoprotein P0). Percent difference was calculated by $2^{-\mathrm{ddCt}}$. The primers (sense and antisense, respectively) were as follows: $36 B 4,5^{\prime}$ - GAG GAA TCA GAT GAG GAT ATG GGA- ${ }^{\prime}$ and $5^{\prime}$ - AAG CAG GCT GAC TTG GTT GC-3'; Scd1, 5' - GAG GCG TGT ACG GGA TCA TA-3', 5'- CAG CGG AGG CTT GTA AGT TC-3'; $S c d 2,5^{\prime}$ - TGT CGC TGA GGT CTG AAG C-3', 5'- TGT GGT GGT GGC TGA GTA AG-3'; Dgat1, 5' - ACG GAT CAT TGA GGG TGT CT-3', 5' - TAG AAC TCG CGG TCT CGA A-3'; Dgat $2,5^{\prime}-$ AGG CGC TAT TTG GCT ACG TT-3', 5' - CAT CAG GTA CTC GGG AAG C-3'; Srebp1c, 5' - ATC TGC TAG AGG GAG CGT TG-3', 5' - TAT TTA GCA ACT GCA GAT ATG CAA G-3'; Lpl, 5' - AGT AGA CTG GTT GTA TCG GG-3', 5' - AGG GTC ATC AGG AGA AAG G-3'; Acc, 5' - AAC ATC CGG ACG CTA AAC AG-3', 5' - CTG ACA AGG TGG CGT GAA G-3'; Fas, 5' - CCC TTG ATG AAG AGG GAT CA-3', 5' - GAA CAA GGG GTT AGG GTT GA-3'; Rip140, 5'- ATG GGT GTT GTC CGT TCG TC-3' ${ }^{\prime}$ 5' - AAC TGG TCG CTC TCT GGT TC-3'; Pgc1a, 5'- ATG TGT CGC CTT CTT GCT CT-3', 5'CAC GAC CTG TGT CGA GAA AA-3'; Prdm16, 5' - CAG CAC GGT GAA GCC ATT C-3', 5'- GGG TGC ATC CGC TTG TG-3'; Bmp7, 5' - CCT GTC CAT CTT AGG GTT GC-3', 5'GCA TTG TAG GGG TAG GAG AAG-3'; Cpt1, 5' - CGA ATC ATC TGG GTG CTG G-3', 5' - AAG AGA CGC CGT AGC CAT CA-3'; $M c d, 5^{\prime}$ - TCG CTG GAT TCA CGA AGT GG-3', 5' - TTC CTC CGA TGC TCG TTC C-3'; Adrb3, 5'-GCT GAC TTG GTA GTG GGA CTC-3' 5' $^{\prime}$ - TAG AAG GAG ACG GAG GAG GAG-3'; Kat, 5' - TGG CAC TCT CTG GGT TGT G-3', 5'- GCA GGT TGT CAC GGT ACT CA-3'; Ucp1, 5'- GAT GGT GAA CGC GAC AAC TT-3', 5' - CTG AAA CTC GGG CTG AGA AG-3'; $m t$ TFA, 5' - AGT TCG CAC GCT GGT AGT GT-3', 5'- GGG GAC ATC TCG ACG C-3'; Niff 1 5' - CAG CAA CGC TGA TGG CAC CGT GTC G-3', 5'- GGC CTC TGA TGG TTG CGT GGT CTG G-3'; llcad, 5' - GAA TGA CGC TGG CAA GAA CGA-3', 5' - ATG CGG ACA ATG TCT GCG AAG-3'; Lcad, 5' - GGA CTC GGG TTC TGG TTC CA3', 5' - TGG AAT CGG GTA GTC CGA CA-3'; Mcad, 5' - CAA CAC TCG AAA GCG GCT CA-3', 5' - ACT TGC GGG CAG TTG CTT G-3'; Fsp27, 5'- GGG CAG TTG CTT CGT TTG TG-3', 5' - AAC ACT GTC TCG CAC ACG TG-3'.

\section{Immunoblot analysis}

Frozen subcutaneous WAT was homogenized in a Tris-EDTA buffer containing $10 \mathrm{mM}$ Tris/HCl $(\mathrm{pH} 7.4)$ and $1 \mathrm{mM}$ EDTA, centrifuged for 10 minutes at $4{ }^{\circ} \mathrm{C}, 800 \mathrm{~g}$ and collected middle layer [25]. Equal amounts $(30 \mu \mathrm{g})$ of proteins were separated on $10 \%$ SDS-polyacrylamide gel and transferred to a nitrocellulose membrane. Primary antibodies used were UCP1 (1:3000), CPT1 (1:1000), FSP27 (1:500), RIP140 (1:1000), PeriA (1:2000), and Actin (1:2000). Actin was used as a loading control. Secondary antibodies were horseradish peroxidase-conjugated anti-rabbit IgG (UCP1, FSP27, RIP140 and PeriA) or anti-goat IgG (CPT1 and Actin). Western blot analysis was performed using Amersham ECL Advance Western Blotting Detection Kit (GE Healthcare, Little Chalfont, UK) and detection was made using a CCDcamera system LAS-4000UVmini (Fujifilm, Tokyo, Japan).

\section{Immunohistochemistry}

Subcutaneous WAT were dissected, fixed, embedded in paraffin, and sectioned. Sections were deparaffinized, treated with $1 \% \mathrm{H}_{2} \mathrm{O}_{2}$ methanol, pre-incubated with $10 \%$ goat serum and exposed to primary antibodies: UCP1 (1:1000), perilipin as positive controls $(1: 100)$. Sections were then incubated with Biotin-conjugated anti-rabbit IgG, treated with peroxidase-conjugated streptavidin, and stained with DAB substrate kit (Nichirei Bioscience, Tokyo, Japan).

\section{Cell culture and transfection}

3T3-L1 preadipocytes were grown in Dulbecco's modified Eagle's medium (DMEM) containing 10\% fetal calf serum (FCS) and seeded on 12-well plates. After reaching confluence, cells were differentiated using $10 \mu \mathrm{g} / \mathrm{ml}$ insulin, $0.5 \mathrm{mM}$ 3-isobutyl-1-methylxanthine, $1 \mu \mathrm{M}$ dexamethasone in DMEM containing $10 \%$ fetal bovine serum (FBS). Following $48 \mathrm{~h}$ of incubation, medium was replaced with DMEM only containing 10\% FBS, and recombinant adenovirus expressing the construct of PeriA was transduced into the cultured cells with LipofectAMINE Plus ${ }^{\text {TM }}$ (Invitrogen, Carlsbad, CA) which protocols were previously described $[9,26,27]$. The human PeriA vector was generated and verified as previously described [13], and various amounts of the vector was transfected to investigate relationships between expressional levels of PeriA and FSP27, and size of lipid droplets [28]. 5 days after transfection, cells were fixed for histology or harvested for immunoblot analysis or quantitative PCR as described above.

\section{Statistical analysis}

Data were analyzed using two-sided Student's $t$-test and significance was set a $p<0.05$. Results are presented as mean values \pm SEM.

\section{Supporting Information}

Figure S1 Oxygen consumption of adipocytes isolated from inguinal WAT of wild-type and PeriA Tg mice. An arrow indicates the addition of $10 \mu \mathrm{M}$ norepinephrine $(\mathrm{NE})$. Data are mean \pm SEM of values from three independent experiments. (177KB TIF) Figure S2 Western blot analysis showing UCP1 protein expression in both BAT and inguinal WAT of WT and Tg mice fed a chow diet. Equal amount $(30 \mu \mathrm{g})$ of proteins was electrophoresed. Actin was used as a loading control. (511KB TIF)

\section{Acknowledgments}

We would like to acknowledge Dr. Mikiko Endo for the generation of PeriA transgenic mice, and Dr. Utano Tomaru at the Department of 
Pathology/Pathophysiology, Hokkaido University Graduate School of Medicine for her support and constructive advice. We also thank research technicians, Aika Ito, Natsumi Fujimori and Asahi Nozawa for their technical support, and Tsutomu Osanai at the Institute for Animal Experimentation, Hokkaido University Graduate School of Medicine for his expertise on procedures involving mice.

\section{References}

1. Kahn R, Buse J, Ferrannini E, Stern M; American Diabetes Association; European Association for the Study of Diabetes (2005) The metabolic syndrome: time for a critical appraisal: joint statement from the American Diabetes Association and the European Association for the Study of Diabetes. Diabetes Care 28: 2289-2304.

2. DeFronzo RA (2004) Dysfunctional fat cells, lipotoxicity and type 2 diabetes. Int J Clin Pract Suppl 143: 9-21.

3. Bickel PE, Tansey JT, Welte MA (2009) PAT proteins, an ancient family of lipid droplet proteins that regulate cellular lipid stores. Biochim Biophys Acta 1791: 419-440.

4. Greenberg AS, Egan JJ, Wek SA, Garty NB, Blanchette-Mackie EJ, et al. (1991) Perilipin, a major hormonally regulated adipocyte-specific phosphoprotein associated with the periphery of lipid storage droplets. J Biol Chem 266: 11341-11346.

5. Brasaemale DL (2007) The perilipin family of structural lipid droplet proteins: stabilization of lipid droplets and control of lipolysis. J Lipid Res 48: 2547-2559.

6. Tansey JT, Sztalryd C, Gruia-Gray J, Roush DL, Zee JV, et al. (2001) Perilipin ablation results in a lean mouse with aberrant adipocyte lipolysis, enhanced leptin production, and resistance to diet-induced obesity. Proc Natl Acad Sci USA 98: 6494 6499.

7. Martinez-Botas J, Anderson JB, Tessier D, Lapillonne A, Chang BH, et al. (2000) Absence of perilipin results in leanness and reverses obesity in Lepr(db/ db) mice. Nat Genet 26: 474-479.

8. Londos C, Sztalryd C, Tanzey JT, Kimmel AR (2005) Role of PAT proteins in lipid metabolism. Biochimie 87: 45-49.

9. Miyoshi H, Souza SC, Zhang HH, Strissel KJ, Christoffolete MA, et al. (2006) Perilipin promotes hormone-sensitive lipase-mediated adipocyte lipolysis via phosphorylation-dependent and -independent mechanisms. J Biol Chem 281: 15837-15844.

10. Puri V, Konda S, Ranjit S, Aouadi M, Chawla A, et al. (2007) Fat-specific protein 27 , a novel lipid droplet protein that enhances triglyceride storage. J Biol Chem 282: 34213-34218.

11. Nishino N, Tamori Y, Tateya S, Kawaguchi T, Shibakusa T, et al. (2008) FSP27 contributes to efficient energy storage in murine white adipocytes by promoting the formation of unilocular lipid droplets. J Clin Invest 118: 2808-21.

12. Toh SY, Gong J, Du G, Li JZ, Yang S, et al. (2008) Up-regulation of mitochondrial activity and acquirement of brown adipose tissue-like property in the white adipose tissue of fsp27 deficient mice. PLoS One 3: e2890.

13. Miyoshi H, Souza SC, Endo M, Sawada T, Perfield JW, 2nd, et al. (2010) Perilipin Overexpression in Mice Protects Against Diet-Induced Obesity. J Lipid Res 51: 975-982.

\section{Author Contributions}

Conceived and designed the experiments: TS HM TK SN NY TK. Performed the experiments: TS KS AS. Analyzed the data: TS KS. Contributed reagents/materials/analysis tools: KS AS YOO JWP CS AG KK. Wrote the paper: TS.

14. Virtanen KA, Lidell ME, Orava J, Heglind M, Westergren R, et al. (2009) Functional brown adipose tissue in healthy adults. N Engl J Med 360: $1518-1525$.

15. Nedergaard J, Cannon B (2010) The changed metabolic world with human brown adipose tissue: therapeutic visions. Cell Metab 11: 268-272.

16. Kahn CR (2008) Can we nip obesity in its vascular bud? Science 322: 542-543.

17. Lazar MA (2008) How now, brown fat? Science 321: 1048-1049.

18. Seale P, Bjork B, Yang W, Kajimura S, Chin S, et al. (2008) PRDM16 controls a brown fat/skeletal muscle switch. Nature 454: 961-968.

19. Tseng YH, Kokkotou E, Schulz TJ, Huang TL, Winnay JN, et al. (2008) New role of bone morphogenic protein 7 in brown adipogenesis and energy expenditure. Nature 454: 1000-1006.

20. Morganstein DL, Christian M, Turner JJ, Parker MG, White R (2008) Conditionally immortalized white preadipocytes: a novel adipocyte model. J Lipid Res 49: 679-685.

21. Hallberg M, Morganstein DL, Kiskinis E, Shah K, Kralli A, et al. (2008) A functional interaction between RIP140 and PGC-1alpha regulates the expression of the lipid droplet protein CIDEA. Mol Cell Biol 28: 6785-6795.

22. Souza SC, Muliro KV, Liscum L, Lien P, Yamamoto MT, et al. (2002) Modulation of hormone-sensitive lipase and protein kinase A-mediated lipolysis by perilipin A in an adenoviral reconstituted system. J Biol Chem 277: 8267-8272.

23. Yoshida T, Sakane N, Umekawa T, Kogure A, Kondo M, et al. (1999) Nicotine induces uncoupling protein 1 in white adipose tissue of obese mice. Int $\mathrm{J}$ Obes Relat Metab Disord 23: 570-575.

24. Livak KJ, Schmittgen TD (2001) Analysis of relative gene expression data using real-time quantitative PCR and the 2(-Delta Delta $\mathrm{C}(\mathrm{T})$ ) method. Methods 25: 402-408.

25. Okamatsu-Ogura Y, Kitao N, Kimura K, Saito M (2007) Brown fat UCP1 is not involved in the febrile and thermogenic responses to IL-1beta in mice. Am J Physiol Endocrinol Metab 292: E1135-E1139.

26. Miyoshi H, Perfield JW, 2nd, Souza SC, Shen WJ, Zhang HH, et al. (2007) Control of ATGL Action by Serine 517 of Perilipin A Globally Regulates PKAstimulated Lipolysis in Adipocytes. J Biol Chem 282: 996-1002.

27. Gauthier MS, Miyoshi H, Souza SC, Cacicedo JM, Saha AK, et al. (2008) AMP-activated Protein Kinase Is Activated as a Consequence of Lipolysis in the Adipocyte: Potential Mechanism and Physiological Relevance. J Biol Chem 283: 16514-16524.

28. Miyoshi H, Perfield JW, 2nd, Obin MS, Greenberg AS (2008) Adipose triglyceride lipase regulates basal lipolysis and lipid droplet size in adipocytes. J Cell Biochem 105: 1430-1436. 